\title{
Complementary feeding indicators and determinants of poor feeding practices in Indonesia: a secondary analysis of 2007 Demographic and Health Survey data
}

\author{
Charmaine $\mathrm{S} \mathrm{Ng}^{1, *}$, Michael J Dibley ${ }^{1}$ and Kingsley E Agho ${ }^{2}$ \\ 'Sydney School of Public Health, Edward Ford Building (A27), University of Sydney, Sydney, NSW 2006, \\ Australia: ${ }^{2}$ School of Medicine, University of Western Sydney, Penrith, Australia
}

Submitted 20 September 2010: Accepted 8 September 2011: First published online 11 October 2011

\begin{abstract}
Objective: The present study aimed to assess complementary feeding practices and identify the potential risk factors associated with inappropriate complementary feeding in Indonesia for a nationally representative sample of births from 2004 to 2007.

Design: The data source for the analysis was the 2007 Indonesia Demographic and Health Survey. Multiple logistic regression was performed to analyse the factors associated with complementary feeding, using individual-, household- and community-level determinants.

Setting: Indonesia.

Subjects: Children ( $n$ 4604) aged 6-23 months.

Results: Multivariate analysis revealed that infants from poor households were significantly less likely to be introduced to complementary feeding (adjusted odds ratio, $\mathrm{AOR}=4 \cdot 32 ; 95 \% \mathrm{CI} 1 \cdot 46,12 \cdot 80)$ and meet the minimum dietary diversity $(\mathrm{AOR}=1 \cdot 76 ; 95 \% \mathrm{CI} 1 \cdot 16,2 \cdot 68)$. Mother's education (AOR for no education in dietary diversity $=1 \cdot 92 ; 95 \%$ CI $1 \cdot 09,3 \cdot 38$; AOR for no education in meal frequency $=2 \cdot 03 ; 95 \%$ CI $1 \cdot 13,3 \cdot 64$; AOR for no education in acceptable diet $=3 \cdot 84 ; 95 \%$ CI $2 \cdot 07,7 \cdot 12$ ), residence and decreased age of the infant were negatively associated with minimum dietary diversity, minimum meal frequency and an acceptable diet. Infants aged 6-11 months were also significantly less likely to meet minimum dietary diversity ( $\mathrm{AOR}=6 \cdot 36$; 95\% CI 4.73, 8.56), minimum meal frequency $(\mathrm{AOR}=2 \cdot 30 ; 95 \% \mathrm{CI} 1 \cdot 79,2 \cdot 96)$ and minimum acceptable diet $(\mathrm{AOR}=2 \cdot 27 ; 95 \% \mathrm{CI} 1 \cdot 67,3 \cdot 09)$. All geographical regions compared with Sumatra were more likely to give the recommended meal frequency and an acceptable diet to breast-fed children.

Conclusions: Public health interventions to improve complementary feeding should address individual-, household- and community-level factors which significantly influence the introduction of complementary feeding. Complementary feeding intervention programmes in Indonesia should ensure that restraints on families with low socio-economic status are addressed. Infants aged 6-11 months and mothers with low education levels may also need special focus. Promotion strategies should also target the health-care delivery system and the media.
\end{abstract}

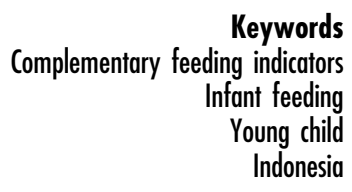

When breast milk alone is no longer nutritionally sufficient for an infant after 6 months of age, the initiation of complementary feeding allows the child to transition gradually to eating family foods. It is estimated that $6 \%$ of deaths among under- $5 \mathrm{~s}$ could be prevented through the achievement of universal coverage with improved complementary feeding alone ${ }^{(1,2)}$. The WHO infant feeding guidelines recommend that infants should be exclusively breast-fed for the first 6 months of life, after which complementary foods may be introduced in conjunction with continued breastfeeding to achieve optimal growth, development and health ${ }^{(3-5)}$. In many developing countries, complementary foods consist of cereals or starchy roots that have been prepared as a thin gruel. Coupled with very few feeds per day, the lack of energy and nutrient content of the complementary foods further increases the risk of child growth retardation and malnutrition ${ }^{(6)}$. With approximately onethird of all children in developing countries under the age of 5 years having low height-for-age and many more being deficient in one or more micronutrients, child undernutrition is a major public health problem in many resource-poor communities in the world ${ }^{(7,8)}$. 
Since the first definitions of infant and young child feeding indicators were proposed by WHO in 1991, there have been important developments in scientific knowledge about what constitutes optimal breast-feeding and complementary feeding practices. In particular, the 1991 infant and young child feeding indicators included only one indicator for complementary feeding - timely complementary feeding. This indicator provided information about the consumption of complementary foods but not about the quantity or quality of those foods. In response to concerns about the lack of adequate indicators of complementary feeding, WHO in 2002 began a process to review and develop new indicators of complementary feeding practices ${ }^{(9)}$. The new indicators of complementary feeding allowed for the calculation of four outcome variables which in turn provided much more comprehensive definitions.

The present paper focuses on the Republic of Indonesia, the fourth most populated country in the world. Despite a majority of all Indonesian children being breast-fed and receiving some form of complementary foods, at the current mortality levels, out of 1000 live births, thirty-one infants die before reaching 5 years of age and $28 \%$ of children in the same age bracket are underweight. With $44 \%$ of all children being stunted and a further $9 \%$ of infants having low birth weight, childhood undernutrition is a significant problem that continues to need to be addressed ${ }^{(10,11)}$.

However, there are few reports about complementary feeding practices within Indonesia, and there are no previous reports examining complementary feeding using the new WHO recommended complementary feeding indicators.

Therefore the present study aims to describe patterns of complementary feeding in Indonesia using the new WHO complementary feeding indicators by analysing the 2007 Indonesia Demographic and Health Survey data. The study also seeks to identify factors associated with inappropriate complementary feeding practices in Indonesia.

\section{Methods}

\section{Data sources}

The data examined were from the 2007 Indonesia Demographic and Health Survey (IDHS), which was conducted in all thirty-three provinces of Indonesia. The survey aimed to gather information about child mortality and maternal and child health, as well as on family planning and other reproductive health issues.

It is a useful and valid source of information on infant and child feeding practices from a representative national household sample of 40701 households. The data collection was based on three main questionnaires: the Household Questionnaire (HQ), the Ever-Married Women's Questionnaire (EMWQ) and the Married Men's Questionnaire (MMQ).

Census blocks were used as the primary sampling unit and the sampling was stratified by urban and rural areas within each province. Systematic random sampling was used to select census blocks followed by a random selection of twenty-five households. Further details of the sampling design and survey methodology are available in the IDHS 2007 report $^{(12)}$.

In this IDHS, 99\% of the 41131 available households were successfully interviewed, and 32895 women were interviewed which comprised $96 \%$ of the 34227 eligible women. Of the 9716 eligible men identified, 8758 were successfully interviewed, yielding a response rate of $90 \%$. The present analysis includes a weighted total (numbers adjusted by the sampling weights) of 4604 children aged 6-23 months.

\section{Conceptual framework}

Figure 1 presents the framework used in the current analysis, along with the selected possible predictors of complementary feeding in Indonesia. The individual-level factors include variables from attributes of the parents, infant and motherinfant dyad. Household environmental factors include the household wealth index and community-level factors include type of residence and geographical region ${ }^{(13,14)}$.

\section{Complementary feeding indicators and explanatory variables}

Complementary feeding practices were assessed using the key indicators recommended by WHO in $2007^{(9)}$, which are based on $24 \mathrm{~h}$ recall data and are defined as follows.

1. Introduction of solid, semi-solid or soft foods: the percentage of infants $6-8$ months of age who received solid, semi-solid or soft foods.

2. Minimum dietary diversity: the percentage of children 6-23 months of age who received foods from four or more food groups. There were only six food groups in the IDHS data instead of seven recommended in the WHO guidelines because eggs and flesh foods were combined as one group. The combined food group of eggs and meat was arbitrarily assigned a weight of two when calculating the dietary diversity index. This is likely to have produced slightly over-optimistic estimates for the minimal dietary diversity index.

3. Minimum meal frequency: the percentage of breastfed and non-breast-fed infants 6-23 months of age who received solid, semi-solid or soft foods the minimum number of times or more. For breast-fed infants aged 6-8 months, the minimum was two times or more during the previous day and this was increased to at least three times for infants aged 9-23 months. For non-breast-fed infants aged 6-23 months, this was established as being fed solid, semi-solid or soft foods for at least four times or more during the previous day.

4. Minimum acceptable diet: the percentage of children 6-23 months of age who received a minimum acceptable diet apart from breast milk. This includes 


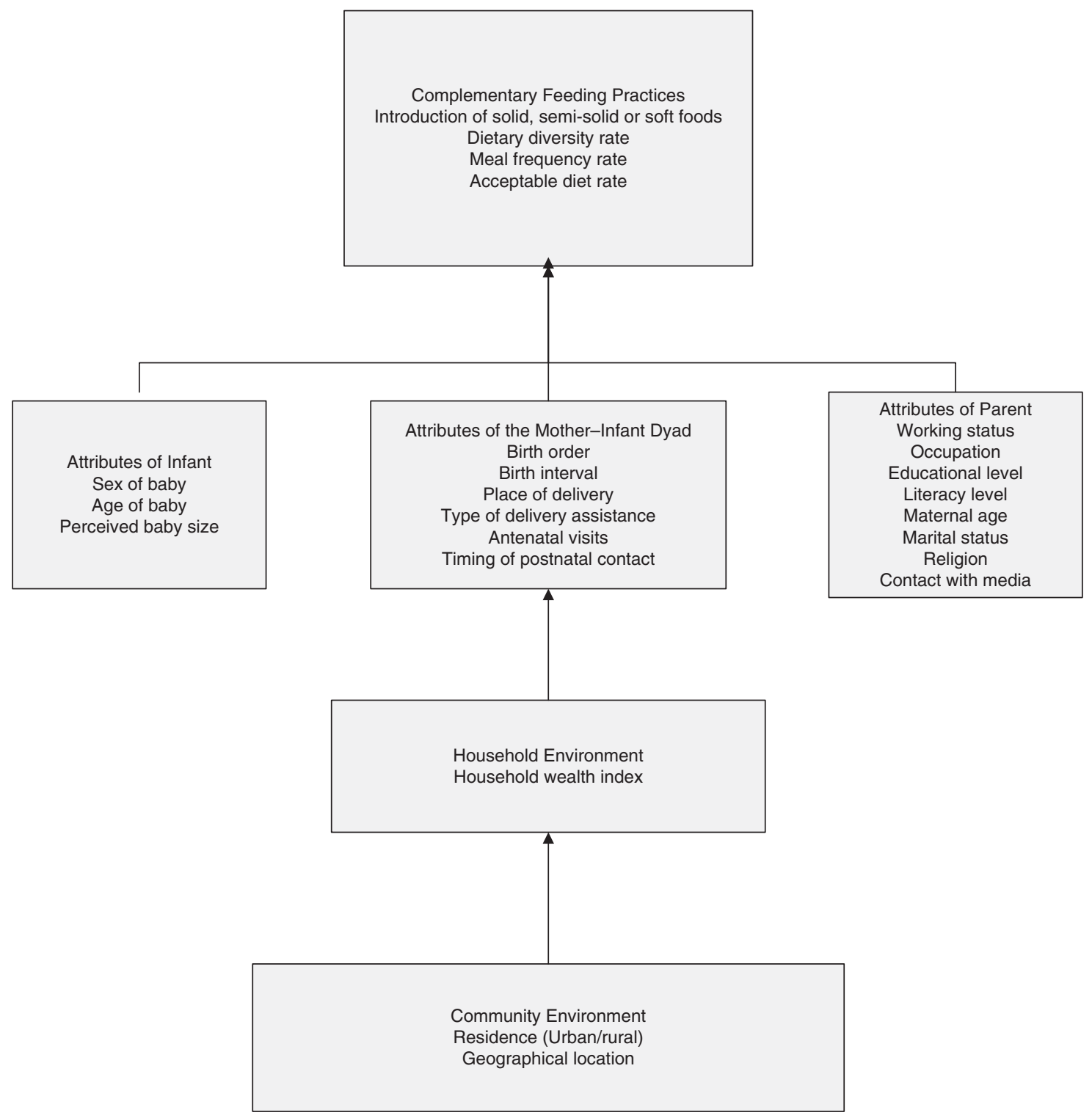

Fig. 1 Conceptual framework of factors influencing complementary feeding practices in Indonesia. Adapted from Mosley and Chen ${ }^{(13)}$

breast-fed and non-breast-fed children 6-23 months of age who had at least the minimum dietary diversity and minimum meal frequency during the previous day with the addition of at least two milk feedings for the non-breast-fed infants. There were no variables available in the IDHS data sets for non-breast-fed children; therefore results presented for this indicator pertain to breast-fed children.

The consumption of Fe-rich or Fe-fortified foods was not examined in this analysis because these data were not collected in IDHS.

Complementary feeding was examined by:

1. individual-level factors, which included mother's working status, father's occupation, mother's working at home or away, mother's education, father's education, mother's literacy, mother's age, mother's marital status, mother's religion, birth order, birth interval, sex of infant, age of child, size of baby, place of delivery, type of delivery assistance, antenatal clinic visits, timing of postnatal check-up, and mother having interaction with the media (newspaper, radio, television);

2. household-level factors, which included the household wealth index; and

3. community-level factors, such as the place of residence and geographical region.

The household wealth index was calculated as score of household assets such as ownership of transportation devices, ownership of durable goods and household 
facilities, which was weighted using the principal components analysis method ${ }^{(15)}$.

\section{Data analysis}

Analyses were confined to the youngest child 6-23 months of age living with the respondent (ever married women aged 15-49 years), although some of the indicators were applicable to different age categories within this range.

The prevalence of infant and young child feeding indicators was estimated across different explanatory variables, and the $\chi^{2}$ test was used to test the statistical significance. Confidence intervals were calculated for prevalence estimates using the survey (SVY) commands to allow for adjustments for the sampling weight and cluster sampling design used in the surveys when estimating confidence intervals around prevalence estimates.

Univariate associations were examined by unadjusted odds ratios for all four selected indicator variables, namely introduction of solid, semi-solid or soft foods, minimum dietary diversity, minimum meal frequency and minimum acceptable diet. Multiple logistic regression analysis was used in a stepwise backward regression model to estimate the adjusted odds ratios and 95\% confidence intervals which allows for identification of the factors significantly associated with complementary feeding practices. Only the variables with statistical significance of $P \leq 0.05$ were retained in the final step of modelling and are presented in the results tables of multivariate analyses. Data analysis was performed using the STATA statistical software package version 10 • (2007; Stata Corporation, College Station, TX, USA).

\section{Results}

\section{Characteristics of the sample}

Table 1 presents the distribution of the individual-, household- and community-level characteristics of 4604 (weighted total) children aged 6-23 months. The sample had a low percentage of working mothers and a very high percentage of literate mothers, with more than half having completed secondary or higher education. More than half of the surveyed mothers had more than one child, and more than half had a preceding birth interval of more than 24 months. According to the mothers' perception of birth size, which has been shown to be a good population-level indicator of birth weight ${ }^{(16,17)}$, about one in seven of the infants was of small size at birth. Of the total births, half were home deliveries and slightly more than half had received trained health professional assistance during delivery. Nearly all mothers had made at least one antenatal clinic visit during their pregnancy.

\section{Complementary feeding indicators}

Of the breast-fed and non-breast-fed children aged 6-23 months, a high percentage $(87 \cdot 3 \%)$ of those aged
6-8 months had been given solid, semi-solid or soft foods (Table 2). The percentage of infants who had received foods from four or more food groups increased with age from $47 \cdot 7 \%$ at $6-11$ months to $84 \cdot 2 \%$ at $18-23$ months. For non-breast-fed children, the percentage meeting the minimum dietary diversity criterion was $60 \cdot 2 \%$ for children aged 6-11 months, $79 \cdot 6 \%$ for children aged $12-17$ months and $88 \cdot 4 \%$ for children aged 18-23 months.

More than half $(53 \cdot 0 \%)$ of infants aged 6-23 months received food 2-4 times or more on the day prior to the survey, but the percentage meeting the minimum meal frequency criterion decreased with increasing age. For non-breast-fed children, the prevalence of minimum meal frequency was $5 \cdot 7 \%$ for children aged 6-11 months, $13.7 \%$ for children aged 12-17 months and $13.5 \%$ for children aged 18-23 months.

The prevalence of minimum acceptable diet in infants aged 6-23 months was only $44.9 \%$, indicating that a greater proportion were either not given food from four or more of the recommended food groups in their diet or the meal frequencies were less than the recommended 2-4 times daily.

\section{Determinants of complementary feeding indicators: univariate analyses}

Table 3 shows the prevalence of complementary feeding indicators across Indonesia and reveals that the percentage of those introduced to solid, semi-solid or soft foods remained relatively high across all the factors examined. There was a significantly lower percentage of introduction of complementary feeds to infants aged 6-8 months for mothers or fathers with no formal education, illiterate or formerly married mothers, no use of antenatal care services or postnatal check-ups, and residence in outer islands especially Kalimantan.

The minimum dietary diversity prevalence was significantly lower in non-working mothers and fathers, including fathers who worked in agriculture, mothers or fathers with no formal education, mothers who were illiterate, younger mothers, mothers of Christian religion, younger infants, home delivered infants, deliveries attended by traditional birth attendants, mothers who did not read newspapers/magazines, listen to the radio or watch television, infants from the poorest households and those who lived in rural areas.

The prevalence of children meeting the minimum meal frequency criterion varied less across the factors examined compared with the other indicators. Minimum meal frequency was significantly lower in mothers with no formal education, illiterate and formerly married mothers, older infants, small sized infants, and in infants from selected geographic areas, especially Sumatra and Kalimantan.

The prevalence of infants meeting the minimum acceptable diet criterion was low in each of the individual-, household- and community-level factors examined. The percentage of infants currently being fed from four or more 
Table 1 Individual-, household- and community-level characteristics of infants 6-23 months of age, Indonesia, 2007 ( $n$ 4604)

\begin{tabular}{|c|c|c|}
\hline Characteristic & $n+$ & $\%$ \\
\hline \multicolumn{3}{|l|}{ Individual-level factors } \\
\hline \multicolumn{3}{|l|}{ Mother's working status ( $n$ 4603) } \\
\hline Non-working & 2765 & $60 \cdot 1$ \\
\hline Working (past 12 months) & 1838 & $39 \cdot 9$ \\
\hline \multicolumn{3}{|l|}{ Father's occupation } \\
\hline Non-agricultural & 3057 & $66 \cdot 4$ \\
\hline Agricultural & 1453 & $31 \cdot 6$ \\
\hline Not working & 94 & $2 \cdot 0$ \\
\hline \multicolumn{3}{|l|}{ Mother works at home or away ( $n$ 1980) } \\
\hline At home & 563 & $28 \cdot 4$ \\
\hline Away & 1417 & $71 \cdot 6$ \\
\hline \multicolumn{3}{|l|}{ Mother's education ( $n$ 4501) } \\
\hline No formal education & 102 & $2 \cdot 2$ \\
\hline Completed primary & 1821 & $39 \cdot 6$ \\
\hline Completed secondary and above & 2680 & $58 \cdot 2$ \\
\hline \multicolumn{3}{|l|}{ Father's education ( $n$ 4593) } \\
\hline No formal education & 105 & $2 \cdot 3$ \\
\hline Completed primary & 1711 & $37 \cdot 3$ \\
\hline Completed secondary and above & 2777 & $60 \cdot 5$ \\
\hline \multicolumn{3}{|l|}{ Mother's literacy ( $n$ 4587) } \\
\hline Cannot read at all & 253 & $5 \cdot 5$ \\
\hline Able to read only part of sentence & 196 & $4 \cdot 3$ \\
\hline Able to read whole sentence & 4138 & $90 \cdot 2$ \\
\hline \multicolumn{3}{|l|}{ Mother's age (years) } \\
\hline $15-24$ & 1346 & $29 \cdot 2$ \\
\hline $25-34$ & 2322 & $50 \cdot 4$ \\
\hline $35-49$ & 936 & $20 \cdot 3$ \\
\hline \multicolumn{3}{|l|}{ Marital status ( $n$ 4603) } \\
\hline Currently married & 4520 & $98 \cdot 2$ \\
\hline \multirow{2}{*}{\multicolumn{3}{|c|}{ Mother's religion }} \\
\hline & & \\
\hline Muslim & 3960 & $86 \cdot 0$ \\
\hline Christian & 525 & $11 \cdot 4$ \\
\hline Other & 119 & $2 \cdot 6$ \\
\hline \multicolumn{3}{|l|}{ Birth order } \\
\hline First-born & 1608 & $34 \cdot 9$ \\
\hline 2nd-4th & 2573 & $55 \cdot 9$ \\
\hline 5th or more & 423 & $9 \cdot 2$ \\
\hline \multicolumn{3}{|l|}{ Preceding birth interval } \\
\hline No previous birth & 1622 & $35 \cdot 2$ \\
\hline$<24$ months & 362 & $7 \cdot 9$ \\
\hline$\geq 24$ months & 2620 & $56 \cdot 9$ \\
\hline \multicolumn{3}{|l|}{ Sex of baby } \\
\hline Male & 2409 & $52 \cdot 3$ \\
\hline Female & 2195 & $47 \cdot 7$ \\
\hline Age of child (months) & & \\
\hline $6-11$ & 1678 & $36 \cdot 5$ \\
\hline $12-17$ & 1499 & $32 \cdot 6$ \\
\hline $18-23$ & 1427 & $31 \cdot 0$ \\
\hline Size of baby ( $n$ 4466) & & \\
\hline Small & 645 & $14 \cdot 4$ \\
\hline Average & 2350 & $52 \cdot 6$ \\
\hline Large & 1471 & $32 \cdot 9$ \\
\hline Place of delivery & & \\
\hline Home & 2322 & $50 \cdot 4$ \\
\hline Health facility & 2282 & $49 \cdot 6$ \\
\hline Type of delivery assistance ( $n$ 4596) & & \\
\hline Health professional & 2551 & $55 \cdot 5$ \\
\hline Traditional birth attendant & 1137 & $24 \cdot 7$ \\
\hline Other untrained & 908 & $19 \cdot 8$ \\
\hline Antenatal clinic visits ( $n$ 4603) & & \\
\hline None & 168 & $3 \cdot 7$ \\
\hline $1-3$ & 639 & $13 \cdot 9$ \\
\hline $4+$ & 3780 & $82 \cdot 1$ \\
\hline Don’t know & 17 & 0.4 \\
\hline Timing of postnatal check-up ( $n$ 4181) & & \\
\hline Immediate (hospital birth) & 2503 & $59 \cdot 9$ \\
\hline Day 1-2 & 834 & $19 \cdot 9$ \\
\hline Day 3-6 & 275 & $6 \cdot 6$ \\
\hline
\end{tabular}

Table 1 Continued

\begin{tabular}{|c|c|c|}
\hline Characteristic & $n+$ & $\%$ \\
\hline Day 7 or later & 89 & $2 \cdot 1$ \\
\hline No check-ups (including missing) & 481 & $11 \cdot 5$ \\
\hline \multicolumn{3}{|c|}{ Reads newspaper or magazine ( $n$ 4592) } \\
\hline Not at all & 2269 & $49 \cdot 4$ \\
\hline Less than once weekly & 1759 & $38 \cdot 3$ \\
\hline At least once weekly & 342 & $7 \cdot 4$ \\
\hline Almost every day & 222 & $4 \cdot 8$ \\
\hline \multicolumn{3}{|l|}{ Listens to radio ( $n$ 4592) } \\
\hline Not at all & 1720 & $37 \cdot 5$ \\
\hline Less than once weekly & 1712 & $37 \cdot 3$ \\
\hline At least once weekly & 338 & $7 \cdot 4$ \\
\hline Almost every day & 822 & $17 \cdot 9$ \\
\hline \multicolumn{3}{|l|}{ Watches television ( $n$ 4595) } \\
\hline Not at all & 357 & $7 \cdot 8$ \\
\hline Less than once weekly & 678 & $14 \cdot 8$ \\
\hline At least once weekly & 261 & $5 \cdot 7$ \\
\hline Almost every day & 3298 & $71 \cdot 8$ \\
\hline \multicolumn{3}{|l|}{ Household-level factors } \\
\hline \multicolumn{3}{|l|}{ Household wealth index } \\
\hline Poorest & 984 & $21 \cdot 4$ \\
\hline Poorer & 837 & $18 \cdot 2$ \\
\hline Middle & 951 & $20 \cdot 7$ \\
\hline Wealthy & 958 & $20 \cdot 8$ \\
\hline Wealthiest & 874 & $19 \cdot 0$ \\
\hline \multicolumn{3}{|l|}{ Community-level factors } \\
\hline \multicolumn{3}{|l|}{ Residence ( $n$ 4603) } \\
\hline Rural & 2699 & $58 \cdot 6$ \\
\hline Urban & 1904 & $41 \cdot 4$ \\
\hline \multicolumn{3}{|l|}{ Geographical region ( $n$ 4339) } \\
\hline Sumatra & 935 & $21 \cdot 6$ \\
\hline Java and Bali & 2493 & $57 \cdot 5$ \\
\hline Eastern part - NTB and NTT & 245 & $5 \cdot 7$ \\
\hline Kalimantan & 303 & $7 \cdot 0$ \\
\hline Sulawesi & 363 & $8 \cdot 4$ \\
\hline
\end{tabular}

NTB, Nusa Tenggara Barat; NTT, Nusa Tenggara Timur. tWeighted total was 4604 unless stated otherwise in parentheses.

food groups, 2-4 times daily was 36.4\% in infants from the 'poorest' wealth index compared with $54.7 \%$ in infants from the 'wealthiest' wealth index. The prevalence of minimum acceptable diet was significantly lower for infants whose parents had a lower level of education, whose mothers did not work, for younger infants, delivered by a traditional birth attendant, whose mother did not attend antenatal care services, whose mothers did not read newspapers/magazines, listen to the radio or watch television, and infants from rural settings.

Within each region, the prevalences of complementary feeding and minimum dietary diversity were better than both the minimum meal frequency and minimum acceptable diet.

\section{Determinants of complementary feeding indicators: multivariate analyses}

Unadjusted and adjusted odds ratios were calculated to estimate the strength of association between independent variables and the four key complementary feeding outcomes: (i) not being introduced to solid, semi-solid or soft foods; (ii) not meeting minimum dietary diversity; (iii) not 
Table 2 Percentage of children who did not reach minimum criteria for complementary feeding indicators among breast-fed and nonbreast-fed children 6-23 months of age, Indonesia, 2007 ( $n$ 4604)

\begin{tabular}{|c|c|c|c|c|}
\hline Indicator & $\begin{array}{c}\text { Sample size, } N \\
\text { (weighted) }\end{array}$ & $\begin{array}{l}\text { Number of children, } n \\
\text { (weighted) }\end{array}$ & $\begin{array}{l}\text { Percentage of } \\
\text { children }\end{array}$ & $95 \% \mathrm{Cl}$ \\
\hline Introduction of solid, semi-solid or soft foods (6-8 months) & 899 & 784 & $87 \cdot 3$ & $83 \cdot 65,90 \cdot 15$ \\
\hline \multicolumn{5}{|l|}{ Minimum dietary diversity +} \\
\hline Minimum dietary diversity (6-11 months) & 1678 & 800 & $47 \cdot 7$ & $44 \cdot 04,51 \cdot 31$ \\
\hline Minimum dietary diversity (12-17 months) & 1499 & 1149 & $76 \cdot 7$ & $73 \cdot 31,79 \cdot 68$ \\
\hline Minimum dietary diversity (18-23 months) & 1427 & 1201 & $84 \cdot 2$ & $80 \cdot 85,86 \cdot 98$ \\
\hline Minimum dietary diversity (6-23 months) & 4604 & 3149 & $68 \cdot 4$ & $66 \cdot 13,70 \cdot 60$ \\
\hline \multicolumn{5}{|l|}{ Minimum meal frequency } \\
\hline Minimum meal frequency (6-11 months) & 1678 & 1046 & $62 \cdot 3$ & $58 \cdot 78,65 \cdot 73$ \\
\hline Minimum meal frequency (12-17 months) & 1499 & 785 & $52 \cdot 4$ & $48 \cdot 39,56 \cdot 33$ \\
\hline Minimum meal frequency (18-23 months) & 1427 & 610 & $42 \cdot 8$ & $38 \cdot 44,47 \cdot 20$ \\
\hline Minimum meal frequency (6-23 months) & 4604 & 2441 & $53 \cdot 0$ & $50 \cdot 75,55 \cdot 27$ \\
\hline \multicolumn{5}{|l|}{ Minimum acceptable diet§,|| } \\
\hline Minimum acceptable diet (6-11 months) & 1434 & 507 & $35 \cdot 4$ & $31 \cdot 73,39 \cdot 14$ \\
\hline Minimum acceptable diet (12-17 months) & 1185 & 584 & $49 \cdot 3$ & $44 \cdot 80,53 \cdot 80$ \\
\hline Minimum acceptable diet (18-23 months) & 815 & 450 & $55 \cdot 2$ & $49 \cdot 36,60 \cdot 91$ \\
\hline Minimum acceptable diet (6-23 months) & 3434 & 1541 & $44 \cdot 9$ & $42 \cdot 17,47 \cdot 60$ \\
\hline
\end{tabular}

tMinimum for dietary diversity: received foods from four or more food groups, consumption of any amount from each food group.

¥Minimum for meal frequency: two meals for breast-fed infants aged 6-8 months, three meals for breast-fed children aged 9-23 months, four meals for nonbreast-fed children aged 6-23 months.

$\S$ Minimum for acceptable diet: combination of dietary diversity and meal frequency.

||Calculated only for breast-fed children.

meeting minimum meal frequency; and (iv) not meeting minimum acceptable diet.

As shown in Table 4, delay in initiating complementary feeding at 6-8 months was significantly associated with decreased age of the child and with a poor household wealth index.

As expected, mother's education had a positive impact on dietary diversity (Table 4). Women from wealthier households or urban areas reported significantly higher dietary diversity than those from poorer households or rural areas. Increasing child's age also had a positive association with dietary diversity. Infants whose mothers read newspapers/magazines and watched television every day were significantly less likely to be fed with four or more food groups. As compared with the Sumatra region, only Kalimantan was significantly more likely to meet minimum dietary diversity criterion.

As seen in Table 4, adequate meal frequency was significantly associated with older age of the child, home delivered or male infants, higher educated mothers and all the regions other than Sumatra. In contrast, meal frequency was significantly lower in women who were divorced, separated or widowed and who had more contact with the media.

Meeting minimum acceptable diet was significantly associated with mothers who were working, mothers with higher levels of education, increasing infant's age, urban residence, and in Kalimantan and Sulawesi.

\section{Discussion}

The present analysis of complementary feeding in Indonesia, which used recent nationally representative data and the new WHO indicators, has revealed that overall the level of the indicators were above $50 \%$ except for the acceptable diet indicator. This is the first report describing complementary feeding indicators in Indonesia using the new WHO indicators.

The study has several strengths. First, the 2007 IDHS was a nationally representative survey using standardised methods that achieved high individual and household response rates. Second, the analyses used the recently recommended WHO infant feeding indicators ${ }^{(9)}$. These new indicators have never been analysed for Indonesia and should help guide the development of appropriate programmes to improve complementary feeding in Indonesia. Finally, the analyses used appropriate adjustments for the complex sampling design of the IDHS.

The study has several potential limitations that should be noted when interpreting the results. First, several variables in the study were not specific to the infants included in the analysis because they reflected only the most recent conditions or birth, such as maternal and paternal occupation, which represented the employment status within the last 12 months preceding the survey. Second, there are limited variables available to measure household- and community-level factors. Third, the crosssectional design of the study means that causal factors for inappropriate complementary feeding could not be established.

\section{Complementary feeding indicators}

Introduction of solid, semi-solid or soft foods

The household wealth index emerged as one of the strongest predictors of poor initiation of solid, semi-solid or soft foods. In a number of studies, household wealth 
Table 3 Percentage of children with appropriate introduction of solid, semi-solid or soft foods, minimum dietary diversity, minimum meal frequency and minimum acceptable diet by individual-, household- and community-level characteristics, Indonesia, 2007

\begin{tabular}{|c|c|c|c|c|c|c|c|c|c|}
\hline \multirow[b]{2}{*}{ Characteristic } & \multirow{2}{*}{$\begin{array}{l}\text { Sample } n \\
\text { (weighted) }\end{array}$} & \multicolumn{2}{|c|}{$\begin{array}{l}\text { Introduction of solid, semi-solid } \\
\text { or soft foods }\end{array}$} & \multicolumn{2}{|c|}{$\begin{array}{l}\text { Minimum dietary } \\
\text { diversity }\end{array}$} & \multicolumn{2}{|c|}{$\begin{array}{l}\text { Minimum meal } \\
\text { frequency }\end{array}$} & \multicolumn{2}{|c|}{$\begin{array}{l}\text { Minimum acceptable } \\
\text { diet ( } 1 \text { year) } \dagger\end{array}$} \\
\hline & & $\%$ & $95 \% \mathrm{Cl}$ & $\%$ & $95 \% \mathrm{Cl}$ & $\%$ & $95 \% \mathrm{Cl}$ & $\%$ & $95 \% \mathrm{Cl}$ \\
\hline \multicolumn{10}{|l|}{ Individual-level factors } \\
\hline \multicolumn{10}{|l|}{ Mother's working status ( $n$ 4603) } \\
\hline Non-working & 2765 & $87 \cdot 5$ & $83 \cdot 05,90 \cdot 92$ & $66 \cdot 5$ & $63 \cdot 64,69 \cdot 26$ & $54 \cdot 4$ & $51 \cdot 31,57 \cdot 48$ & $42 \cdot 2$ & $38 \cdot 89,45 \cdot 67$ \\
\hline Working (past 12 months) & 1838 & $86 \cdot 7$ & $80 \cdot 15,91 \cdot 35$ & $71 \cdot 3^{*}$ & $67 \cdot 88,74 \cdot 45$ & $50 \cdot 9$ & $47 \cdot 67,54 \cdot 16$ & $49 \cdot 3^{*}$ & $45 \cdot 10,53 \cdot 43$ \\
\hline \multicolumn{10}{|l|}{ Father's occupation } \\
\hline Non-agricultural & 3057 & $91 \cdot 0$ & $87 \cdot 22,93 \cdot 81$ & $71 \cdot 6$ & $68 \cdot 67,74 \cdot 27$ & $53 \cdot 3$ & $50 \cdot 44,56 \cdot 18$ & $47 \cdot 0$ & $43 \cdot 48,50 \cdot 48$ \\
\hline Agricultural & 1453 & $80 \cdot 7$ & $72 \cdot 85,86 \cdot 61$ & $62 \cdot 1$ & $58 \cdot 49,65 \cdot 49$ & $53 \cdot 0$ & $49 \cdot 45,56 \cdot 49$ & $41 \cdot 0$ & $36 \cdot 90,45 \cdot 22$ \\
\hline Not working & 94 & $51 \cdot 0^{\star \star}$ & $21 \cdot 13,80 \cdot 12$ & $64 \cdot 4^{\star \star}$ & $50 \cdot 70,76 \cdot 01$ & $43 \cdot 8$ & $30 \cdot 05,58 \cdot 53$ & $43 \cdot 4$ & $25 \cdot 98,62 \cdot 57$ \\
\hline \multicolumn{10}{|l|}{ Mother works at home or away ( $n 1980)$} \\
\hline At home & 563 & $85 \cdot 0$ & $66 \cdot 39,94 \cdot 18$ & $70 \cdot 8$ & $64 \cdot 29,76 \cdot 58$ & $51 \cdot 9$ & $46 \cdot 31,57 \cdot 50$ & $46 \cdot 9$ & $38 \cdot 79,55 \cdot 16$ \\
\hline Away & 1417 & $89 \cdot 4$ & $83 \cdot 68,93 \cdot 24$ & $70 \cdot 8$ & $67 \cdot 18,74 \cdot 21$ & $52 \cdot 3$ & $48 \cdot 48,56 \cdot 00$ & $49 \cdot 7^{\star}$ & $45 \cdot 38,54 \cdot 11$ \\
\hline \multicolumn{10}{|l|}{ Mother's education ( $n$ 4501) } \\
\hline No formal education & 102 & $65 \cdot 8$ & $29 \cdot 11,90 \cdot 02$ & $46 \cdot 4$ & $33 \cdot 68,59 \cdot 66$ & $40 \cdot 6$ & $29 \cdot 83,52 \cdot 35$ & $22 \cdot 3$ & $14 \cdot 01,33 \cdot 45$ \\
\hline Completed primary & 1821 & $84 \cdot 2$ & $77 \cdot 14,89 \cdot 39$ & $65 \cdot 8$ & $62 \cdot 27,69 \cdot 13$ & $54 \cdot 0$ & $50 \cdot 38,57 \cdot 55$ & $40 \cdot 7$ & $36 \cdot 69,44 \cdot 85$ \\
\hline Completed secondary and above & 2680 & $89 \cdot 7$ & $85 \cdot 39,92 \cdot 84$ & $71 \cdot 0^{\star *}$ & $68 \cdot 15,73 \cdot 73$ & $52 \cdot 8$ & $49 \cdot 99,55 \cdot 65$ & $49 \cdot 1^{\star *}$ & $45 \cdot 69,52 \cdot 60$ \\
\hline \multicolumn{10}{|l|}{ Father's education ( $n$ 4593) } \\
\hline No formal education & 105 & $73 \cdot 9$ & $50 \cdot 71,88 \cdot 60$ & $43 \cdot 7$ & $32 \cdot 12,55 \cdot 91$ & $52 \cdot 1$ & $40 \cdot 42,63 \cdot 58$ & $31 \cdot 6$ & $20 \cdot 90,44 \cdot 57$ \\
\hline Completed primary & 1711 & $86 \cdot 8$ & $79 \cdot 84,91 \cdot 64$ & $64 \cdot 9$ & $61 \cdot 50,68 \cdot 14$ & $53 \cdot 3$ & $49 \cdot 54,56 \cdot 97$ & $38 \cdot 6$ & $34 \cdot 46,42 \cdot 97$ \\
\hline Completed secondary and above & 2777 & $88 \cdot 2$ & $83 \cdot 43,91 \cdot 66$ & $71 \cdot 6^{\star \star}$ & $68 \cdot 76,74 \cdot 28$ & $52 \cdot 9$ & $50 \cdot 08,55 \cdot 62$ & $49 \cdot 7^{\star \star}$ & $46 \cdot 37,53 \cdot 10$ \\
\hline \multicolumn{10}{|l|}{ Mother's literacy ( $n$ 4587) } \\
\hline Cannot read at all & 253 & $78 \cdot 2$ & $59 \cdot 02,89 \cdot 97$ & $54 \cdot 7$ & $45 \cdot 62,63 \cdot 39$ & $46 \cdot 0$ & $37 \cdot 79,54 \cdot 49$ & $29 \cdot 5$ & $21 \cdot 82,38 \cdot 51$ \\
\hline Able to read only part of sentence & 196 & $96 \cdot 6$ & $88 \cdot 40,99 \cdot 07$ & $64 \cdot 1$ & $53 \cdot 91,73 \cdot 14$ & $55 \cdot 5$ & $46 \cdot 18,64 \cdot 41$ & $45 \cdot 5$ & $34 \cdot 28,57 \cdot 27$ \\
\hline \multirow{2}{*}{\multicolumn{10}{|c|}{ Mother's age (years) }} \\
\hline & & & & & & & & & \\
\hline $15-24$ & 1346 & $86 \cdot 9$ & $79 \cdot 06,92 \cdot 11$ & $64 \cdot 5$ & $59 \cdot 98,68 \cdot 84$ & $53 \cdot 6$ & $49 \cdot 77,57 \cdot 46$ & $41 \cdot 8$ & $37 \cdot 08,46 \cdot 85$ \\
\hline $25-34$ & 2322 & $86 \cdot 1$ & $81 \cdot 02,89 \cdot 96$ & $71 \cdot 0$ & $68.38,73.53$ & $51 \cdot 4$ & $48 \cdot 43,54 \cdot 43$ & $47 \cdot 0$ & $43 \cdot 54,50 \cdot 49$ \\
\hline $35-49$ & 936 & $90 \cdot 7$ & $82 \cdot 15,95 \cdot 37$ & $67 \cdot 5^{\star}$ & $62 \cdot 77,71 \cdot 89$ & $56 \cdot 1$ & $51 \cdot 24,60 \cdot 80$ & $44 \cdot 1$ & $38 \cdot 36,49 \cdot 98$ \\
\hline \multicolumn{10}{|l|}{ Marital status ( $n$ 4603) } \\
\hline Currently married & 4520 & $87 \cdot 5$ & $83 \cdot 88,90 \cdot 41$ & $68 \cdot 5$ & $66 \cdot 17,70 \cdot 64$ & $53 \cdot 3$ & $51 \cdot 06,55 \cdot 61$ & $44 \cdot 9$ & $42 \cdot 20,47 \cdot 68$ \\
\hline Formerly married (divorced/separated/widow) & 83 & $63 \cdot 3$ & $28 \cdot 85,88 \cdot 03$ & $66 \cdot 1$ & $50 \cdot 02,79 \cdot 19$ & $35 \cdot 6^{*}$ & $23 \cdot 60,49 \cdot 68$ & $40 \cdot 2$ & $24 \cdot 61,58 \cdot 15$ \\
\hline \multicolumn{10}{|l|}{ Mother's religion } \\
\hline Muslim & 3960 & $88 \cdot 1$ & $83 \cdot 94,91 \cdot 25$ & $69 \cdot 1$ & $66 \cdot 62,71 \cdot 41$ & $53 \cdot 2$ & $50 \cdot 63,55 \cdot 75$ & $44 \cdot 9$ & $41.94,47 \cdot 92$ \\
\hline Christian & 525 & $80 \cdot 9$ & $72 \cdot 12,87 \cdot 44$ & $63 \cdot 6$ & $57 \cdot 82,68 \cdot 92$ & $51 \cdot 1$ & $46 \cdot 56,55 \cdot 58$ & $43 \cdot 1$ & $36 \cdot 68,49 \cdot 73$ \\
\hline Other & 119 & $88 \cdot 8$ & $76 \cdot 53,95 \cdot 06$ & $67 \cdot 9$ & $58 \cdot 68,75 \cdot 85$ & $55 \cdot 7$ & $47 \cdot 98,63 \cdot 12$ & $51 \cdot 3$ & $42 \cdot 34,60 \cdot 16$ \\
\hline \multicolumn{10}{|l|}{ Birth order } \\
\hline First-born & 1608 & $86 \cdot 9$ & $79 \cdot 00,92 \cdot 11$ & $68 \cdot 1$ & $63 \cdot 89,71 \cdot 95$ & $52 \cdot 7$ & $48 \cdot 81,56 \cdot 51$ & $47 \cdot 5$ & $42 \cdot 69,52 \cdot 44$ \\
\hline 2nd-4th & 2573 & $87 \cdot 5$ & $82 \cdot 97,91 \cdot 02$ & $69 \cdot 3$ & $66 \cdot 46,72 \cdot 00$ & $52 \cdot 6$ & $49 \cdot 71,55 \cdot 48$ & $44 \cdot 3$ & $40 \cdot 84,47 \cdot 71$ \\
\hline 5th or more & 423 & $86 \cdot 7$ & $76 \cdot 31,92 \cdot 91$ & $64 \cdot 3$ & $57 \cdot 72,70 \cdot 43$ & $56 \cdot 9$ & $51 \cdot 03,62 \cdot 49$ & $40 \cdot 0$ & $33 \cdot 31,47 \cdot 00$ \\
\hline \multicolumn{10}{|l|}{ Preceding birth interval } \\
\hline No previous birth & 1622 & $87 \cdot 2$ & $79 \cdot 39,92 \cdot 28$ & $68 \cdot 0$ & $63 \cdot 87,71 \cdot 90$ & $52 \cdot 7$ & $48 \cdot 83,56 \cdot 50$ & $47 \cdot 5$ & $42 \cdot 62,52 \cdot 34$ \\
\hline$<24$ months & 362 & $81 \cdot 4$ & $69 \cdot 23,89 \cdot 54$ & $69 \cdot 3$ & $62 \cdot 41,75 \cdot 37$ & $51 \cdot 9$ & $44 \cdot 91,58 \cdot 88$ & $43 \cdot 1$ & $34 \cdot 88,51 \cdot 71$ \\
\hline$\geq 24$ months & 2620 & $88 \cdot 1$ & $83 \cdot 78,91 \cdot 43$ & $68 \cdot 5$ & $65 \cdot 75,71 \cdot 18$ & $53 \cdot 4$ & $50 \cdot 46,56 \cdot 28$ & $43 \cdot 7$ & $40 \cdot 42,47 \cdot 03$ \\
\hline \multicolumn{10}{|l|}{ Sex of baby } \\
\hline Male & 2409 & $87 \cdot 1$ & $82 \cdot 09,90 \cdot 91$ & $69 \cdot 4$ & $66 \cdot 58,72 \cdot 08$ & $55 \cdot 1$ & $52 \cdot 07,58 \cdot 11$ & $47 \cdot 1$ & $43 \cdot 37,50 \cdot 82$ \\
\hline Female & 2195 & $87 \cdot 4$ & $81 \cdot 95,91 \cdot 35$ & $67 \cdot 3$ & $63.80,70 \cdot 65$ & $50 \cdot 7$ & $47 \cdot 55,53 \cdot 90$ & $42 \cdot 5$ & $38 \cdot 83,46 \cdot 29$ \\
\hline
\end{tabular}




\begin{tabular}{|c|c|c|c|c|c|c|c|c|c|}
\hline \multirow[b]{2}{*}{ Characteristic } & \multirow{2}{*}{$\begin{array}{l}\text { Sample } n \\
\text { (weighted) }\end{array}$} & \multicolumn{2}{|c|}{$\begin{array}{l}\text { Introduction of solid, semi-solid } \\
\text { or soft foods }\end{array}$} & \multicolumn{2}{|c|}{$\begin{array}{l}\text { Minimum dietary } \\
\text { diversity }\end{array}$} & \multicolumn{2}{|c|}{$\begin{array}{l}\text { Minimum meal } \\
\text { frequency }\end{array}$} & \multicolumn{2}{|c|}{$\begin{array}{l}\text { Minimum acceptable } \\
\text { diet ( } 1 \text { year) } \dagger\end{array}$} \\
\hline & & $\%$ & $95 \% \mathrm{Cl}$ & $\%$ & $95 \% \mathrm{Cl}$ & $\%$ & $95 \% \mathrm{Cl}$ & $\%$ & $95 \% \mathrm{Cl}$ \\
\hline \multicolumn{10}{|l|}{ Age of child (months) } \\
\hline $6-11$ & 1678 & & & $47 \cdot 7$ & $44 \cdot 03,51 \cdot 32$ & $62 \cdot 3$ & $58 \cdot 75,65 \cdot 76$ & $35 \cdot 4$ & $31 \cdot 71,39 \cdot 16$ \\
\hline $12-17$ & 1499 & & & $76 \cdot 7$ & $73 \cdot 31,79 \cdot 68$ & $52 \cdot 4$ & $48 \cdot 39,56 \cdot 33$ & $49 \cdot 3$ & $44 \cdot 78,53 \cdot 82$ \\
\hline $18-23$ & 1427 & & & $84 \cdot 2^{\star \star}$ & $80 \cdot 83,86 \cdot 99$ & $42 \cdot 8^{\star *}$ & $38 \cdot 43,47 \cdot 21$ & $55 \cdot 2^{\star \star}$ & $49 \cdot 36,60 \cdot 91$ \\
\hline \multicolumn{10}{|l|}{ Size of baby $(n 4466)$} \\
\hline Small & 645 & $81 \cdot 8$ & $67 \cdot 34,90 \cdot 73$ & $64 \cdot 2$ & $58 \cdot 34,69 \cdot 61$ & $48 \cdot 4$ & $42 \cdot 44,54 \cdot 36$ & $40 \cdot 1$ & $33 \cdot 68,46 \cdot 84$ \\
\hline Average & 2350 & $89 \cdot 6$ & $84 \cdot 67,93 \cdot 07$ & $68 \cdot 8$ & $65 \cdot 69,71 \cdot 81$ & $53 \cdot 7$ & $50 \cdot 65,56 \cdot 68$ & $44 \cdot 8$ & $41 \cdot 16,48 \cdot 56$ \\
\hline Large & 1471 & $86 \cdot 1$ & $79 \cdot 80,90 \cdot 64$ & $71 \cdot 2^{*}$ & $67 \cdot 64,74 \cdot 58$ & $53 \cdot 5$ & $49 \cdot 69,57 \cdot 28$ & $47 \cdot 6$ & $43 \cdot 04,52 \cdot 19$ \\
\hline \multicolumn{10}{|l|}{ Place of delivery } \\
\hline Home & 2322 & $84 \cdot 4$ & $79 \cdot 51,88 \cdot 30$ & $66 \cdot 0$ & $63 \cdot 12,68 \cdot 80$ & $54 \cdot 6$ & $51 \cdot 49,57 \cdot 76$ & $43 \cdot 5$ & $39 \cdot 88,47 \cdot 14$ \\
\hline Health facility & 2282 & $90 \cdot 1$ & $84 \cdot 30,93 \cdot 87$ & $70 \cdot 8^{*}$ & $67 \cdot 44,74 \cdot 02$ & $51 \cdot 4$ & $48 \cdot 14,54 \cdot 59$ & $46 \cdot 5$ & $42 \cdot 66,50 \cdot 34$ \\
\hline \multicolumn{10}{|l|}{ Type of delivery assistance ( $n$ 4596) } \\
\hline Health professional & 2551 & $89 \cdot 6$ & $84 \cdot 58,93 \cdot 18$ & $71 \cdot 1$ & $68 \cdot 06,73 \cdot 88$ & $52 \cdot 4$ & $49 \cdot 55,55 \cdot 25$ & $47 \cdot 4$ & $43.98,50 \cdot 80$ \\
\hline Traditional birth attendant & 1137 & $83 \cdot 5$ & $75 \cdot 62,89 \cdot 21$ & $65 \cdot 0$ & $60 \cdot 80,68 \cdot 95$ & $52 \cdot 5$ & $47 \cdot 88,57 \cdot 10$ & $39 \cdot 4$ & $34 \cdot 61,44 \cdot 30$ \\
\hline Other untrained & 908 & $84 \cdot 0$ & $76 \cdot 71,89 \cdot 33$ & $65 \cdot 5^{\star}$ & $60 \cdot 36,70 \cdot 25$ & $55 \cdot 5$ & $50 \cdot 45,60 \cdot 42$ & $45 \cdot 8^{*}$ & $39 \cdot 91,51 \cdot 87$ \\
\hline \multicolumn{10}{|l|}{ Antenatal clinic visits ( $n$ 4603) } \\
\hline None & 168 & $78 \cdot 2$ & $60 \cdot 91,89 \cdot 15$ & $56 \cdot 4$ & $47 \cdot 07,65 \cdot 34$ & $45 \cdot 7$ & $37 \cdot 10,54 \cdot 51$ & $32 \cdot 9$ & $24 \cdot 25,42 \cdot 94$ \\
\hline $1-3$ & 639 & $79 \cdot 7$ & $67 \cdot 93,87 \cdot 91$ & $62 \cdot 8$ & $56 \cdot 94,68 \cdot 37$ & $55 \cdot 4$ & $50 \cdot 36,60 \cdot 34$ & $40 \cdot 4$ & $34 \cdot 11,46 \cdot 99$ \\
\hline $4+$ & 3780 & $89 \cdot 0$ & $85 \cdot 14,91 \cdot 88$ & $69 \cdot 9$ & $67 \cdot 38,72 \cdot 29$ & $53 \cdot 1$ & $50 \cdot 53,55 \cdot 60$ & $46 \cdot 3$ & $43 \cdot 26,49 \cdot 41$ \\
\hline Don't know & 17 & $74 \cdot 3^{*}$ & $22 \cdot 44,96 \cdot 67$ & $65 \cdot 3^{*}$ & $46 \cdot 61,80 \cdot 27$ & $25 \cdot 1$ & $13 \cdot 13,42 \cdot 57$ & $28 \cdot 4^{*}$ & $12 \cdot 75,51 \cdot 86$ \\
\hline \multicolumn{10}{|l|}{ Timing of postnatal check-up ( $n$ 4181) } \\
\hline Immediate (hospital birth) & 2503 & $89 \cdot 0$ & $84 \cdot 10,92 \cdot 47$ & $68 \cdot 4$ & $65 \cdot 25,71 \cdot 39$ & $53 \cdot 5$ & $50 \cdot 36,56 \cdot 58$ & $43 \cdot 6$ & $40 \cdot 04,47 \cdot 24$ \\
\hline Day 1-2 & 834 & $88 \cdot 2$ & $79 \cdot 34,93 \cdot 58$ & $69 \cdot 6$ & $64 \cdot 69,74 \cdot 01$ & $53 \cdot 6$ & $48 \cdot 55,58 \cdot 49$ & $49 \cdot 0$ & $43 \cdot 31,54 \cdot 67$ \\
\hline Day 3-6 & 275 & $84 \cdot 1$ & $62 \cdot 07,94 \cdot 44$ & $65 \cdot 4$ & $55 \cdot 25,74 \cdot 39$ & $55 \cdot 7$ & $46 \cdot 68,64 \cdot 35$ & $41 \cdot 5$ & $31 \cdot 86,51 \cdot 84$ \\
\hline Day 7 or later & 89 & $82 \cdot 9$ & $46 \cdot 97,96 \cdot 37$ & $75 \cdot 9$ & $61 \cdot 02,86 \cdot 39$ & $51 \cdot 5$ & $35 \cdot 59,67 \cdot 09$ & $53 \cdot 7$ & $33 \cdot 67,72 \cdot 52$ \\
\hline No check-ups (including missing) & 481 & $75 \cdot 2$ & $63 \cdot 62,84.08$ & $62 \cdot 4$ & $56 \cdot 67,67 \cdot 79$ & $48 \cdot 7$ & $43 \cdot 88,53.56$ & $39 \cdot 3$ & $33 \cdot 82,45 \cdot 12$ \\
\hline \multicolumn{10}{|c|}{ Reads newspaper or magazine ( $n$ 4592) } \\
\hline Not at all & 2269 & $87 \cdot 3$ & $82 \cdot 10,91 \cdot 14$ & $63 \cdot 5$ & $60 \cdot 22,66 \cdot 64$ & $52 \cdot 9$ & $49 \cdot 69,56 \cdot 14$ & $39 \cdot 6$ & $35 \cdot 98,43 \cdot 27$ \\
\hline Less than once weekly & 1759 & $85 \cdot 6$ & $78 \cdot 69,90 \cdot 48$ & $71 \cdot 3$ & $68 \cdot 01,74 \cdot 39$ & $55 \cdot 2$ & $51 \cdot 64,58 \cdot 73$ & $49 \cdot 6$ & $45 \cdot 33,53 \cdot 77$ \\
\hline At least once weekly & 342 & $86 \cdot 8$ & $75 \cdot 38,93 \cdot 43$ & $78 \cdot 5$ & $71 \cdot 95,83.90$ & $43 \cdot 7$ & $37 \cdot 00,50 \cdot 68$ & $50 \cdot 0$ & $41 \cdot 27,58 \cdot 67$ \\
\hline Almost every day & 222 & $98 \cdot 7$ & $93 \cdot 44,99 \cdot 77$ & $80 \cdot 2^{\star \star}$ & $71 \cdot 86,86 \cdot 53$ & $51 \cdot 7$ & $43 \cdot 07,60 \cdot 31$ & $61 \cdot 7^{\star \star}$ & $50 \cdot 34,71 \cdot 95$ \\
\hline \multicolumn{10}{|l|}{ Listens to radio ( $n$ 4592) } \\
\hline Not at all & 1720 & $84 \cdot 5$ & $77 \cdot 56,89 \cdot 62$ & $64 \cdot 0$ & $60 \cdot 55,67 \cdot 30$ & $51 \cdot 5$ & $47 \cdot 86,55 \cdot 16$ & $40 \cdot 0$ & $36 \cdot 04,44 \cdot 07$ \\
\hline Less than once weekly & 1712 & $86 \cdot 6$ & $79 \cdot 98,91 \cdot 21$ & $69 \cdot 6$ & $65 \cdot 86,73 \cdot 12$ & $52 \cdot 6$ & $49 \cdot 06,56 \cdot 14$ & $47 \cdot 6$ & $43 \cdot 20,52 \cdot 01$ \\
\hline At least once weekly & 338 & $91 \cdot 6$ & $80 \cdot 73,96 \cdot 62$ & $76 \cdot 1$ & $69 \cdot 08,81 \cdot 95$ & $58 \cdot 3$ & $50 \cdot 66,65 \cdot 52$ & $52 \cdot 7$ & $43 \cdot 36,61 \cdot 79$ \\
\hline Almost every day & 822 & $92 \cdot 0$ & $84 \cdot 90,95 \cdot 95$ & $72 \cdot 1^{*}$ & $66 \cdot 95,76 \cdot 78$ & $54 \cdot 7$ & $49 \cdot 70,59 \cdot 70$ & $46 \cdot 8^{*}$ & $40 \cdot 87,52 \cdot 81$ \\
\hline \multicolumn{10}{|l|}{ Watches television ( $n$ 4595) } \\
\hline Not at all & 357 & $88 \cdot 3$ & $79 \cdot 91,93 \cdot 49$ & $50 \cdot 0$ & $42 \cdot 70,57 \cdot 24$ & $56 \cdot 1$ & $49 \cdot 28,62 \cdot 60$ & $33 \cdot 6$ & $26 \cdot 30,41 \cdot 73$ \\
\hline Less than once weekly & 678 & $86 \cdot 9$ & $78 \cdot 47,92 \cdot 40$ & $64 \cdot 2$ & $58 \cdot 61,69 \cdot 49$ & $54 \cdot 4$ & $49 \cdot 19,59 \cdot 44$ & $41 \cdot 3$ & $35 \cdot 21,47 \cdot 75$ \\
\hline At least once weekly & 261 & $90 \cdot 7$ & $78 \cdot 20,96 \cdot 34$ & $65 \cdot 5$ & $57 \cdot 33,72 \cdot 80$ & $52 \cdot 7$ & $41 \cdot 09,57 \cdot 32$ & $38 \cdot 0$ & $29 \cdot 63,47 \cdot 20$ \\
\hline Almost every day & 3298 & $86 \cdot 9$ & $82 \cdot 07,90 \cdot 61$ & $71 \cdot 5^{\star \star}$ & $69 \cdot 12,73 \cdot 74$ & $52 \cdot 7$ & $49 \cdot 98,55 \cdot 41$ & $47 \cdot 6^{*}$ & $44.48,50 \cdot 67$ \\
\hline \multicolumn{10}{|l|}{ Household-level factors } \\
\hline \multicolumn{10}{|l|}{ Household wealth index } \\
\hline Poorest & 984 & $80 \cdot 0$ & $72 \cdot 00,86 \cdot 21$ & $57 \cdot 4$ & $53 \cdot 19,61 \cdot 43$ & $52 \cdot 6$ & $48 \cdot 84,56 \cdot 30$ & $36 \cdot 4$ & $32 \cdot 18,40 \cdot 75$ \\
\hline Poorer & 837 & $85 \cdot 6$ & $75 \cdot 84,91 \cdot 79$ & $67 \cdot 0$ & $61 \cdot 82,71 \cdot 70$ & $52 \cdot 8$ & $48 \cdot 24,57 \cdot 29$ & $42 \cdot 2$ & $37 \cdot 10,47 \cdot 52$ \\
\hline Middle & 951 & $89 \cdot 9$ & $81 \cdot 45,94 \cdot 77$ & $69 \cdot 8$ & $64 \cdot 81,74 \cdot 29$ & $54 \cdot 2$ & $48 \cdot 67,59 \cdot 63$ & $46 \cdot 9$ & $40 \cdot 82,53 \cdot 05$ \\
\hline Wealthy & 958 & $87 \cdot 1$ & $76 \cdot 59,93 \cdot 32$ & $70 \cdot 7$ & $65 \cdot 03,75 \cdot 71$ & $54 \cdot 2$ & $48 \cdot 92,59 \cdot 32$ & $47 \cdot 1$ & $40 \cdot 89,53 \cdot 46$ \\
\hline Wealthiest & 874 & $95 \cdot 3$ & $87 \cdot 89,98 \cdot 24$ & $78 \cdot 3^{\star \star}$ & $73 \cdot 62,82 \cdot 36$ & $51 \cdot 2$ & $45 \cdot 96,56 \cdot 41$ & $54 \cdot 7^{\star \star}$ & $48 \cdot 09,61 \cdot 10$ \\
\hline
\end{tabular}


index was related to poor infant feeding and was closely associated with low birth weight and stunting ${ }^{(18,19)}$. Shorter birth intervals of less than 12 months have been reported in earlier literature as a strong predictor of poor infant feeding ${ }^{(20-24)}$. This could be related to maternal depletion syndrome and resource competition between siblings, in addition to a lack of care and attention experienced by first-born infants ${ }^{(25-28)}$.

\section{Dietary diversity}

A close relationship was observed between the utilisation of media such as newspapers, magazines and television and a lower prevalence of adequate dietary diversity. However, this result suggests that the mass media has the potential to promote dietary diversity, educating and advising parents or caregivers of the benefits of serving a variety of foods. A project undertaken in Indonesia in 1985, which drew on the mass media to improve child feeding practices at home, led to much improvement in infant feeding knowledge and practices among mothers ${ }^{(29)}$. It is established that educational interventions can lead to improved feeding practices ${ }^{(30)}$. In Peru, a community-based controlled efficacy study assessed the impact of regular nutrition advice in the home on dietary intake in infants $7-11$ months of age. The results showed that complementary food intake, and thus intakes of energy and nutrients, increased during the short study period. This was achieved through increasing consumption of certain food groups and recommended food preparations, including foods from animal sources ${ }^{(31,32)}$.

At the household level, high wealth index demonstrated a significant association with dietary diversity. There was a progressive increase in the prevalence of children meeting the dietary diversity criterion as wealth index increased. Although most mothers or caregivers were aware that dietary diversity plays an important role in the child's health, this knowledge-practice discrepancy appears to imply that lack of resources acts as a barrier for mothers in their efforts to put knowledge into practice ${ }^{(33)}$.

The geographic differences in dietary diversity were significant in the multivariate analysis, with infants living in Java and Bali less likely to be given four or more food groups than those living in Sumatra. This might relate to different food cultures or beliefs about the variety of foods that are suitable for infants or the availability of foods.

\section{Meal frequency and acceptable diet}

Feeding patterns such as the timing and number of meals or snacks require close attention ${ }^{(2)}$. One of the independent variables likely to interact with the intake of complementary foods is the behaviour of the caregiver, which includes the level of encouragement provided to the child during feeding, the frequency the food is being fed and the environment where feeding takes place ${ }^{(2,34-36)}$. 
Table 4 Survey logistic modelling of a child not currently receiving adequate complementary feeding (unadjusted and adjusted odds ratios), Indonesia, 2007

\begin{tabular}{|c|c|c|c|c|c|c|c|}
\hline \multirow[b]{2}{*}{ Outcome variable } & \multirow[b]{2}{*}{ Characteristic } & \multicolumn{3}{|c|}{ Unadjusted } & \multicolumn{3}{|c|}{ Adjusted } \\
\hline & & OR & $95 \% \mathrm{Cl}$ & $P$ & OR & $95 \% \mathrm{Cl}$ & $P$ \\
\hline \multirow[t]{4}{*}{ Not being introduced to solid, semi-solid or soft foods } & Age of child (months) & 0.57 & $0 \cdot 39,0 \cdot 84$ & 0.004 & $0 \cdot 56$ & $0 \cdot 38,0 \cdot 81$ & 0.002 \\
\hline & Household wealth index & 1.00 & & & & & \\
\hline & $\begin{array}{l}\text { Rich } \\
\text { Middle }\end{array}$ & $\begin{array}{l}1 \cdot 00 \\
2 \cdot 39\end{array}$ & & & $1 \cdot 00$ & & \\
\hline & Poor & 3.97 & $0 \cdot 75,7 \cdot 57$ & $0 \cdot 287$ & $\begin{array}{l}2 \cdot 57 \\
4 \cdot 32\end{array}$ & $0 \cdot 83,8 \cdot 01$ & $\begin{array}{l}0 \cdot 103 \\
0.008\end{array}$ \\
\hline \multirow[t]{26}{*}{ Not meeting minimum dietary diversity } & Mother's education & & 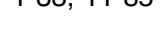 & 000 & & & \\
\hline & Completed secondary and above & $1 \cdot 00$ & & & $1 \cdot 00$ & & \\
\hline & Completed primary & $1 \cdot 27$ & $1 \cdot 03,1 \cdot 57$ & 0.024 & $1 \cdot 05$ & $0 \cdot 81,1 \cdot 37$ & $0 \cdot 709$ \\
\hline & No education & $2 \cdot 76$ & $1 \cdot 48,5 \cdot 15$ & $0 \cdot 001$ & $1 \cdot 92$ & $1 \cdot 09,3 \cdot 38$ & 0.023 \\
\hline & Age of child (months) & & & & & & \\
\hline & $18-23$ & $1 \cdot 00$ & & & $1 \cdot 00$ & & \\
\hline & $12-17$ & $1 \cdot 65$ & $1 \cdot 21,2 \cdot 24$ & 0.002 & $1 \cdot 68$ & $1 \cdot 23,2 \cdot 29$ & 0.001 \\
\hline & $6-11$ & $5 \cdot 92$ & $4 \cdot 41,7 \cdot 95$ & $<0.001$ & $6 \cdot 36$ & $4 \cdot 73,8 \cdot 56$ & $<0.001$ \\
\hline & Reads newspaper or magazine & & & & & & \\
\hline & Not at all/less than once weekly/at least once weekly & $1 \cdot 00$ & & & $1 \cdot 00$ & & \\
\hline & Almost every day & 1.92 & $1 \cdot 41,2 \cdot 61$ & $<0.001$ & 1.53 & $1 \cdot 10,2 \cdot 11$ & 0.011 \\
\hline & Watches television & & & & & & \\
\hline & Not at all/less than once weekly/at least once weekly & 1.00 & & & & & \\
\hline & Almost every day & $1 \cdot 69$ & $1 \cdot 37,2 \cdot 09$ & $<0.001$ & $1 \cdot 36$ & $1 \cdot 06,1 \cdot 74$ & $0 \cdot 015$ \\
\hline & $\begin{array}{l}\text { Household wealth index } \\
\text { Rich }\end{array}$ & $1 \cdot 00$ & & & 1.00 & & \\
\hline & Middle & 1.53 & $1 \cdot 14,2 \cdot 07$ & 0.005 & $1 \cdot 37$ & $0.97,1.95$ & 0.073 \\
\hline & Poor & $2 \cdot 18$ & $1 \cdot 61,2 \cdot 95$ & $<0.001$ & $1 \cdot 76$ & $1 \cdot 16,2 \cdot 68$ & 0.008 \\
\hline & Residence & & & & & & \\
\hline & Urban & $1 \cdot 00$ & & & $1 \cdot 00$ & & \\
\hline & Rural & $1 \cdot 54$ & $1 \cdot 22,1 \cdot 96$ & $<0.001$ & $1 \cdot 34$ & $1 \cdot 00,1 \cdot 78$ & 0.047 \\
\hline & Geographical region & & & & & & \\
\hline & Sumatra & $1 \cdot 00$ & & & $1 \cdot 00$ & & \\
\hline & Java and Bali & $1 \cdot 17$ & $0.90,1.51$ & 0.234 & $1 \cdot 42$ & $1 \cdot 08,1 \cdot 86$ & 0.013 \\
\hline & Eastern part - NTB and NTT & $1 \cdot 47$ & $1 \cdot 04,2 \cdot 09$ & 0.030 & $1 \cdot 33$ & $0.91,1.93$ & $0 \cdot 138$ \\
\hline & Kalimantan & 1.06 & $0 \cdot 80,1 \cdot 40$ & 0.686 & $0 \cdot 96$ & $0 \cdot 72,1 \cdot 27$ & 0.754 \\
\hline & Sulawesi & 1.09 & $0.83,1.43$ & 0.536 & $1 \cdot 01$ & $0.75,1.36$ & 0.949 \\
\hline \multirow{14}{*}{ Not meeting minimum meal frequency } & Mother's education & & & & & & \\
\hline & Completed secondary and above & $1 \cdot 00$ & & & $1 \cdot 00$ & & \\
\hline & Completed primary & 0.97 & $0 \cdot 80,1 \cdot 17$ & 0.750 & $1 \cdot 05$ & $0 \cdot 85,1 \cdot 28$ & 0.673 \\
\hline & No education & $1 \cdot 75$ & $1.01,3.06$ & 0.047 & $2 \cdot 03$ & $1 \cdot 13,3 \cdot 64$ & 0.017 \\
\hline & Marital status & & & & & & \\
\hline & Currently married & $1 \cdot 00$ & & & $1 \cdot 00$ & & \\
\hline & Formerly married (divorced/separated/widow) & $2 \cdot 14$ & $1 \cdot 16,3 \cdot 97$ & $0 \cdot 015$ & $2 \cdot 12$ & $1 \cdot 16,3 \cdot 87$ & 0.014 \\
\hline & Sex of baby & & & & & & \\
\hline & Male & 1.00 & & & $1 \cdot 00$ & & \\
\hline & Female & $1 \cdot 21$ & $1 \cdot 01,1 \cdot 45$ & 0.042 & $1 \cdot 24$ & $1 \cdot 04,1 \cdot 49$ & 0.019 \\
\hline & Age of child (months) & & & & & & \\
\hline & $18-23$ & $1 \cdot 00$ & & & $1 \cdot 00$ & & \\
\hline & $12-17$ & $1 \cdot 52$ & $1 \cdot 20,1 \cdot 93$ & $<0.001$ & $1 \cdot 55$ & $1 \cdot 22,1 \cdot 98$ & $<0.001$ \\
\hline & $6-11$ & $2 \cdot 24$ & $1 \cdot 74,2 \cdot 86$ & $<0.001$ & $2 \cdot 30$ & $1 \cdot 79,2.96$ & $<0.001$ \\
\hline
\end{tabular}


Table 4 Continued

\begin{tabular}{|c|c|c|c|c|c|c|c|}
\hline \multirow[b]{2}{*}{ Outcome variable } & \multirow[b]{2}{*}{ Characteristic } & \multicolumn{3}{|c|}{ Unadjusted } & \multicolumn{3}{|c|}{ Adjusted } \\
\hline & & OR & $95 \% \mathrm{Cl}$ & $P$ & OR & $95 \% \mathrm{Cl}$ & $P$ \\
\hline & Place of delivery & & & & & & \\
\hline & Home & $1 \cdot 00$ & & & $1 \cdot 00$ & & \\
\hline & $\begin{array}{l}\text { Health facility } \\
\text { Reads newspaner or magazine }\end{array}$ & $1 \cdot 13$ & $0.94,1 \cdot 37$ & 0.202 & $1 \cdot 25$ & $1 \cdot 00,1 \cdot 56$ & 0.047 \\
\hline & Not at all/less than once weekly/at least once weekly & $1 \cdot 00$ & & & $1 \cdot 00$ & & \\
\hline & Almost every day & $1 \cdot 33$ & $1 \cdot 04,1 \cdot 70$ & 0.025 & $1 \cdot 36$ & $1 \cdot 05,1 \cdot 75$ & 0.018 \\
\hline & Listens to radio & & & & & & \\
\hline & Not at all/less than once weekly/qt least once weekly & $1 \cdot 00$ & & & $1 \cdot 00$ & & \\
\hline & $\begin{array}{l}\text { Almost every day } \\
\text { Geographical region }\end{array}$ & $1 \cdot 15$ & $0.92,1 \cdot 44$ & $0 \cdot 216$ & $1 \cdot 27$ & $1 \cdot 01,1 \cdot 60$ & 0.044 \\
\hline & Sumatra & $1 \cdot 00$ & & & $1 \cdot 00$ & & \\
\hline & Java and Bali & 0.66 & $0.54,0.81$ & $<0.001$ & 0.63 & $0.51,0.78$ & 0.001 \\
\hline & Eastern part - NTB and NTT & $0 \cdot 70$ & $0.50,0.99$ & 0.043 & $0 \cdot 70$ & $0.50,0.99$ & 0.041 \\
\hline & Kalimantan & $0 \cdot 84$ & $0.65,1.08$ & $0 \cdot 167$ & 0.85 & $0 \cdot 65,1 \cdot 11$ & 0.234 \\
\hline & Sulawesi & 0.66 & $0.52,0.83$ & 0.001 & 0.66 & $0.52,0.85$ & 0.001 \\
\hline \multirow[t]{19}{*}{ Not meeting minimum acceptable diet } & Mother's working status & & & & & & \\
\hline & Working (past 12 months) & $1 \cdot 00$ & & & $1 \cdot 00$ & & \\
\hline & Non-working & $1 \cdot 34$ & $1 \cdot 07,1 \cdot 67$ & 0.010 & $1 \cdot 34$ & $1 \cdot 07,1 \cdot 67$ & 0.012 \\
\hline & Mother's education & & & & & & \\
\hline & Completed secondary and above & $1 \cdot 00$ & & & $1 \cdot 00$ & & \\
\hline & Completed primary & $1 \cdot 41$ & $1 \cdot 13,1 \cdot 75$ & 0.003 & $1 \cdot 42$ & $1 \cdot 12,1 \cdot 79$ & 0.003 \\
\hline & No education & $3 \cdot 80$ & $2 \cdot 01,7 \cdot 16$ & $<0.001$ & $3 \cdot 84$ & $2 \cdot 07,7 \cdot 12$ & $<0.001$ \\
\hline & Age of child (months) & & & & & & \\
\hline & $18-23$ & $1 \cdot 00$ & & & $1 \cdot 00$ & & \\
\hline & $12-17$ & $1 \cdot 29$ & $0.94,1.77$ & $0 \cdot 119$ & $1 \cdot 30$ & $0.95,1.79$ & 0.097 \\
\hline & $\begin{array}{l}\text { 6-11 } \\
\text { Residence }\end{array}$ & $2 \cdot 25$ & $1 \cdot 66,3 \cdot 07$ & $<0.001$ & $2 \cdot 27$ & $1 \cdot 67,3 \cdot 09$ & $<0.001$ \\
\hline & Urban & $1 \cdot 00$ & & & $1 \cdot 00$ & & \\
\hline & Rural & $1 \cdot 47$ & $1 \cdot 15,1 \cdot 87$ & 0.002 & $1 \cdot 46$ & $1 \cdot 13,1 \cdot 89$ & 0.004 \\
\hline & Geographical region & & & & & & \\
\hline & Sumatra & $1 \cdot 00$ & & & $1 \cdot 00$ & & \\
\hline & Java and Bali & 0.89 & $0 \cdot 69,1 \cdot 15$ & 0.379 & 0.90 & $0 \cdot 69,1 \cdot 16$ & 0.407 \\
\hline & Eastern part - NTB and NTT & 0.98 & $0 \cdot 70,1 \cdot 38$ & 0.905 & $0 \cdot 87$ & $0.62,1 \cdot 24$ & 0.453 \\
\hline & Kalimantan & $0 \cdot 75$ & $0.56,1.00$ & 0.053 & 0.68 & $0.50,0.91$ & 0.011 \\
\hline & Sulawesi & 0.73 & $0.55,0.96$ & 0.024 & 0.65 & $0.48,0.86$ & 0.003 \\
\hline
\end{tabular}

NTB, Nusa Tenggara Barat; NTT, Nusa Tenggara Timur.

The categories of explanatory variables have been ordered by placing the category with the lowest risk of the adverse feeding pattern first and this sequence may vary from earlier tables. 
Mothers who gave birth in a health facility had a poor association with meal frequency. This is a contradictory finding, with further examination of the data also revealing that the prevalence of bottle-feeding was higher in women who delivered in health facilities $(42.9 \%)$ than in those who delivered at home $(24 \cdot 2 \%)$. This can be explained by wealthier women, who were more likely to bottle-feed, giving birth in health facilities, thus explaining the lower prevalence of adequate meal frequency. This also indicates that women delivering in health facilities may not have been appropriately counselled or given advice about infant feeding, suggesting a need to improve the delivery of infant and young child feeding messages in health facilities.

Mothers who were divorced, separated or widowed also had a negative association with meeting the meal frequency criterion. Studies have shown that single mothers lack support from families or communities, which causes poor infant feeding practices ${ }^{(37)}$. This indicates that health institutions and health-care professionals can play a significant role in promoting complementary feeding. Evidence ${ }^{(36)}$ shows that infant feeding counselling in health facilities is effective, not only to improve breast-feeding practices, but also to improve complementary feeding. In one population where the duration of breast-feeding was typically short and where complementary foods were introduced early, the provision of counselling in health facilities was attributed to a decline in growth faltering among children older than 6 months. Through improvements to maternal complementary feeding knowledge, increases in energy and nutrient intakes were observed and were qualitatively demonstrated through improved feeding practices such as the timely introduction of complementary foods and the quality of such foods ${ }^{(38)}$. Similar findings were observed in a large community-based study in Haryana, India where complementary feeding practices were improved through the provision of appropriate information and support to families and caregivers through the health system and the community ${ }^{(39)}$.

However, mother's education demonstrated a significant association with both meal frequency and the acceptable diet criterion. The present analysis revealed that more educated mothers were more likely to feed their child 2-4 times or more on the previous day, which allows higher likelihood of the child consuming at least one animal-source food and at least one fruit or vegetable that day. Similar findings associating infant feeding with education have also been described in studies undertaken in Uganda and Pakistan ${ }^{(18,40)}$. Higher-educated mothers were associated with more beneficial feeding practices than their less-educated peers, indicating that nutrition and feeding guidance for mothers needs to be especially targeted to lower-educated mothers ${ }^{(41)}$.

The prevalences of adequate meal frequency and acceptable diet were both shown to be positively significant within all geographic regions.

\section{Conclusions}

The importance of improving appropriate complementary feeding practices is paramount in reducing undernutrition and excess morbidity and mortality in children in developing countries. There is an urgent need for a collective effort to achieve the Millennium Development Goals for the prevention of undernutrition and to improve child survival. Furthermore, infant feeding recommendations need to be adapted to the specific settings in which they are to be implemented. Previous research has shown that complementary feeding can be improved and one possible method in achieving this goal is through promotion programmes that provide counselling and education to mothers regarding appropriate foods and frequency of feeding of young children.

Most complementary feeding indicators in Indonesia could be improved in order to gain the full benefits of appropriate complementary feeding in reducing undernutrition, morbidity and mortality in young children.

\section{Acknowledgements}

This research received no specific grant from any funding agency in the public, commercial or not-for-profit sectors. This analysis is part of the first author's dissertation to fulfil the requirements for a Master of International Public Health with Honours at the University of Sydney. There are no conflicts of interest. C.S.N. and M.J.D. designed the study; C.S.N. performed the analysis and prepared the manuscript; M.J.D. and K.E.A. provided data analysis advice. M.J.D. provided revision of the final manuscript. All authors read and approved the manuscript. C.S.N. would like to thank Associate Professor Michael J Dibley for helping with the study design and Dr Kingsley Agho for help with the analysis.

\section{References}

1. United Nations Standing Committee on Nutrition (2003) Meeting the challenge to improve complementary feeding. SCN News 27, 1-42.

2. Lutter C (2003) Meeting the challenge to improve complementary feeding. SCN News 27, 4-9.

3. World Health Organization (2003) Global Strategy on Infant and Young Child Feeding. Geneva: WHO.

4. World Health Organization (2007) Planning Guide for National Implementation of the Global Strategy for Infant and Young Child Feeding. Geneva: WHO.

5. World Health Organization (2008) The International Code of Marketing of Breast-Milk Substitutes - Frequently Asked Questions. Geneva: WHO.

6. Gibson R, Ferguson E \& Lehrfeld J (1998) Complementary foods for infant feeding in developing countries: their nutrient adequacy and improvement. Eur J Clin Nutr 52, 764-770.

7. Black RE, Allen LH, Bhutta ZA et al. (2008) Maternal and child undernutrition: global and regional exposures and health consequences. Lancet 371, 243-260. 
8. Ruel MT, Brown KH \& Caulfield LE (2003) Moving forward with complementary feeding: indicators and research priorities. International Food Policy Research Institute (IFPRI) discussion paper 146 (April 2003). Food Nutr Bull 24, 289-290.

9. World Health Organization, UNICEF, United States Agency for International Development, Academy for Educational Development, University of California Davis \& International Food Policy Research Institute (2008) Indicators for Assessing Infant and Young Child Feeding Practices (Part 1 Definitions): Conclusions of a Consensus Meeting held 6-8 November 2007 in Washington D.C., USA. Geneva: WHO.

10. UNICEF (2004) At a glance: Indonesia. http://www.unicef. org/infobycountry/indonesia_statistics.html (accessed August 2009).

11. World Food Programme (2009) Countries: Indonesia. http:// www.wfp.org/countries/indonesia (accessed June 2009).

12. Statistics Indonesia (Badan Pusat Statistik - BPS) \& Macro International (2008) Indonesia Demographic and Health Survey 2007 [National Family Planning Coordinating Board, Ministry of Heath and ORC Macro, editors] Calverton, MD: BPS and ORC Macro International.

13. Mosley WH \& Chen LC (1984) An analytical framework for the study of child survival in developing countries. Popul Dev Rev 10, Suppl., 25-45.

14. Hector D, King L \& Webb K (2005) Factors affecting breastfeeding practices applying a conceptual framework. NSW Public Health Bull 16, 52-55.

15. Filmer D \& Pritchett L (2001) Estimating wealth effects without expenditure data - or tears: an application to educational enrollments in states of India. Demography 38, 115-132.

16. Boerma J, Weinstein K, Rutstein S et al. (1996) Data on birth weight in developing countries: can surveys help? Bull World Health Organ 74, 209-216.

17. Mbuagbaw L \& Gofin R (2010) Can recall of birth size be used as a measure of birthweight in Cameroon? Paediatr Perinat Epidemiol 24, 383-389.

18. Fadnes LT, Engebretsen IM, Wamani H et al. (2009) Infant feeding among HIV-positive mothers and the general population mothers: comparison of two cross-sectional surveys in Eastern Uganda. BMC Public Health 9, 124.

19. Engebretsen IM, Tylleskär T, Wamani H et al. (2008) Determinants of infant growth in Eastern Uganda: a community-based cross-sectional study. BMC Public Health 8, 418.

20. Naylor AJ \& Morrow AL (editors) (2001) Developmental Readiness of Normal Full Term Infants to Progress from Exclusive Breastfeeding to the Introduction of Complementary Foods: Reviews of the Relevant Literature Concerning Infant Immunologic, Gastrointestinal, Oral Motor and Maternal Reproductive and Lactational Development. Washington, DC: Wellstart International and the LINKAGES Project/Academy for Educational Development.

21. Molbak K, Gottschau A, Aaby P et al. (1994) Prolonged breast feeding, diarrhoeal disease, and survival of children in Guinea-Bissau. BMJ 308, 1403-1406.

22. WHO Collaborative Study Team on the Role of Breastfeeding on the Prevention of Infant Mortality (2000) Effect of breastfeeding on infant and child mortality due to infectious diseases in less developed countries: a pooled analysis. Lancet 355, 451-455.

23. Texas Department of State Health Services (1994) Infant Feeding: Low Birth Weight and Preterm Infants. Austin, TX: Texas Department of State Health Services.
24. Pan American Health Organization \& World Health Organization (2004) Guiding Principles for Complementary Feeding of the Breastfed Child. Geneva: WHO.

25. Bushra F, Ahmad N, Hussain T et al. (1995) Effect of infant feeding practices on birth interval and morbidity among children in north west frontier province of Pakistan. J Islamic Acad Sci 8, 69-72.

26. United Nations Standing Committee on Nutrition (2009) Thirty-First Session of the Standing Committee on Nutrition SCN Working Group on Breastfeeding and Complementary Feeding: Contribution to Millennium Development Goals (MDGs). http://www.unscn.org/en/home/contact-us.php (accessed September 2009).

27. Rutstein SO (2005) Effects of preceding birth intervals on neonatal, infant and under-five years mortality and nutritional status in developing countries: evidence from the demographic and health surveys. Int J Gynaecol Obstet 89, Suppl. 1, S7-S24.

28. Zenger E (1993) Siblings' neonatal mortality risks and birth spacing in Bangladesh. Demography 30, 477-488.

29. The Manoff Group (1992) Improving Young Child Feeding Practices Project Final Report. Washington, DC: The Manoff Group.

30. Bhandari $\mathrm{N}$, Mazumder S, Bahl $\mathrm{R}$ et al. (2004) An educational intervention to promote appropriate complementary feeding practices and physical growth in infants and young children in rural Haryana, India. J Nutr 134, 2342-2348.

31. Daelmans B \& Saadeh R (2003) Global initiatives to improve complementary feeding. SCN News 27, 10-18.

32. Sellen DW (2001) Weaning, complementary feeding, and maternal decision making in rural East African pastoral population. J Hum Lact 17, 233-244.

33. Wamani $\mathrm{H}$, Astrøm AN, Peterson $\mathrm{S}$ et al. (2005) Infant and young child feeding in western Uganda: knowledge, practices and socio-economic correlates. $J$ Trop Pediatr 51, 356-361.

34. Dewey KG \& Brown KH (2003) Update on technical issues concerning complementary feeding of young children in developing countries and implications for intervention programs. Food Nutr Bull 24, 5-26.

35. Pelto GH, Levitt E \& Thairu L (2003) Improving feeding practices: current patterns, common constraints, and the design of interventions. Food Nutr Bull 24, 45-82.

36. Daelmans B, Martines J \& Saadeh R (2003) Conclusions of the global consultation on complementary feeding. Food Nutr Bull 24, 126-129.

37. World Health Organization Country Office for India (2008) MCH Community Newsletter Breastfeeding Month Special. Geneva: WHO.

38. Santos I, Victora CG, Martines J et al. (2001) Nutrition counseling increases weight gain among Brazilian children. J Nutr 131, 2866-2873.

39. Bhandari N, Bahl R, Mazumdar S et al. (2003) Effect of community-based promotion of exclusive breastfeeding on diarrhoeal illness and growth: a cluster randomised controlled trial. Lancet 361, 1418-1423.

40. Liaqat P, Rizvi MA, Qayyum A et al. (2006) Maternal education and complementary feeding. Pakistan J Nutr $\mathbf{5}$, 563-568.

41. Fein SB, Labiner-Wolfe J, Scanlon KS et al. (2008) Selected complementary feeding practices and their association with maternal education. Pediatrics 122, Suppl. 2, S91-S97. 